\title{
Capability of a Portable Chromatic Unit for Monitoring Airborne Particles over Wide Urban Areas
}

\author{
M. A. Aceves-Fernandez, ${ }^{1,2}$ G. R. Jones, ${ }^{1}$ Y. R. Kolupula, ${ }^{1}$ T. Reichelt, ${ }^{1}$ J. W. Spencer, ${ }^{1}$ \\ C. Pedraza-Ortega, ${ }^{2}$ and E. Gorrostieta ${ }^{2}$ \\ ${ }^{1}$ Centre for Intelligent Monitoring Systems (CIMS), Department of Electrical Engineering and Electronics, The University of Liverpool, \\ Brownlow Hill, Liverpool L69 3GJ, UK \\ ${ }^{2}$ Centre for Research and Development on Informatics and Telecommunications (CIDIT), Faculty of Informatics, \\ Universidad Autónoma de Querétaro, Campus Juriquilla, Querétaro 76230, Mexico \\ Correspondence should be addressed to M. A. Aceves-Fernandez, marco.aceves@uaq.mx
}

Received 8 May 2009; Revised 29 June 2009; Accepted 3 August 2009

Recommended by Cristina E. Davis

Investigations are described into the use of a compact, portable unit, using polychromatic scattered light, for the preliminary monitoring airborne PM 2-10 particulates over widespread urban areas subjected to vehicular traffic. The monitoring unit has been used at a city centre bus terminus, at sites along an urban bus corridor and outside a local school adjacent to an air quality monitoring station. Holistic results are presented which demonstrate the use of the unit for the preliminary identification of locations and conditions with elevated levels of PM 2-10 particles which can be investigated in greater detail with particle-specific, high-precision instruments.

Copyright (c) 2009 M. A. Aceves-Fernandez et al. This is an open access article distributed under the Creative Commons Attribution License, which permits unrestricted use, distribution, and reproduction in any medium, provided the original work is properly cited.

\section{Introduction}

Monitoring airborne PM2.5/PM10 particulates in urban/ suburban environments (e.g., [1-3]) is important for environmental and health reasons. There are several sources of such particulates, some of which are exhaust emissions, tyre wear, and mechanical operation of road vehicles including buses [4-6]. Investigating levels, and so forth of particulates from such sources over a breadth of real operational conditions is difficult in practice, particularly if disruption of the normal operation of the transport system is to be avoided. The use of separate units, each devoted to a particular source (e.g., a separate unit mounted on each exhaust of individual vehicles), is uneconomic. Also widespread usage of fixed site precision instruments (e.g., particle micro weighing (TEOM) [7], laser scattering [8], "black smoke" optics [9], etc.) is prohibitively expensive or yielding only limited information. However a prototype system based upon the chromatic detection of polychromatic light scattering described by Reichelt et al. [10] has the potential for satisfying such needs and has already been used in the semi benign environment of the void at a bus terminus. The unit was intended for conveniently obtaining fingerprints of airborne micro particles over wide urban areas in a holistic manner rather than as an instrument for the accurate measurement of specific particles and their concentrations.

This paper is concerned with the evaluation of an adaptation of the prototype unit described by Reichelt et al. [10] to form a portable, robust and cost effective unit for field use. The intention was to employ it in a holistic manner for obtaining approximate trends in the occurrence and variations in the concentration of airborne micro particles in the range $2-10$ microns so that conditions and locations warranting more detailed measurements with precision instruments could be identified. The unit has been deployed for monitoring such PM2-PM10 particles at sites along an urban bus corridor, on the busy apron of a city centre bus terminus during the arrival and departure of buses and outside a local school adjacent to a DEFRA air quality monitoring station. These areas of deployment were more demanding than the secluded and protected environment of 


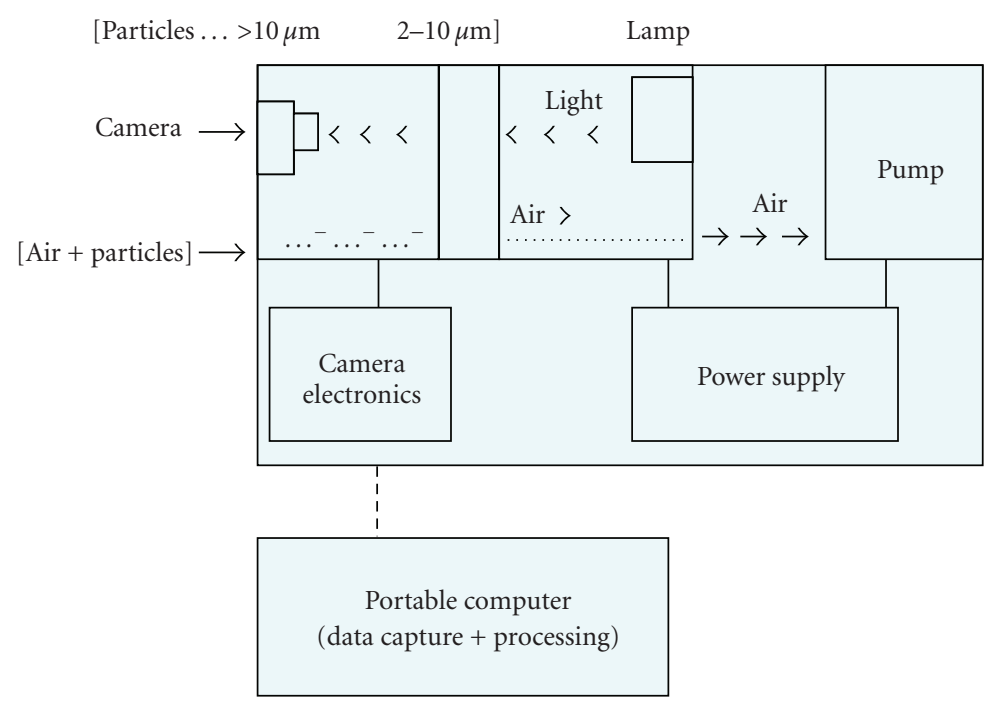

(a)

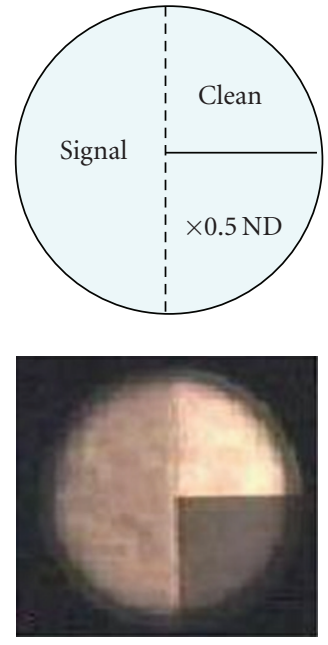

(b)

FIgure 1: Chromatic particle monitoring system: (a) Schematic Diagram of the Portable Unit, (b) Structure of CCD Image of the Particle Accumulator $(\times 0.5 \mathrm{ND}=\times 0.5$ Neutral Density Optical Filter $)$.

the void enclosure monitored by Reichelt et al. [10]. It is shown how the outputs from the portable instrument may be benchmarked during a sequence of routine tests at several sites against outputs from the DEFRA air quality monitoring station. Details of the deployment of the instrument along with examples of the results and information obtained from such tests are described.

\section{Monitoring Technique}

The monitoring technique used a miniature CCD camera for tracking changes in the spectral properties of polychromatic light scattered from microparticles accumulated, from an airflow, onto a micro pore particle filter [10]. The measurement procedure involved chromatically processing the scattered polychromatic light $[11,12]$ using the outputs from the $R, G$, $B$ pixels of the CCD camera to yield values for the chromatic parameters, Hue $(\mathrm{H}$, dominant wavelength), Lightness (L, signal strength), and Saturation ( $\mathrm{S}$, spectral spread) (e.g., $[13,14])$ using the algorithms given in the appendix. The values of these parameters were empirically calibrated against particle concentrations as described by Reichelt et al. [10].

A schematic diagram of the portable particulates monitoring unit used for the present tests is shown on Figure 1(a). It consisted of a tubular enclosure through which samples of air were drawn by a miniature pump at a flow rate of 3 or $10 \mathrm{~L} / \mathrm{min}$. By varying the flow rate, the number of particles drawn in a given time from a fixed airborne concentration could be altered, so controlling the operational range of the monitor. The air was first drawn through a filter, which prevented particulates of size greater than PM10 from entering the duct, whilst a second micropore filter within the duct trapped the 2 to $10 \mu \mathrm{m}$ particles. This glass microfibre filter was backlit with white light from a tungsten halogen source and viewed directly with the miniature CCD camera, which captured images of the filter and particles. The portable unit also contained a battery driven power supply (for the miniature air flow pump, the light source, and the CCD camera) and voltage control electronics for varying the pump speed and the light intensity provided by the tungsten halogen source. The output signals from the camera were fed directly into a portable computer for storage and processing. The particle monitoring unit was compact, lightweight, and convenient to use in field trials.

The use of the CCD camera as the sensing element provided a convenient means for cross checking automatically the authenticity of the captured optical signals, which was important for the portable and robust use of the system under the harsh environments of the present tests. Figure 1(b) shows a schematic diagram of the image of the back-lit micro-particle filter captured by the CCD camera. The image consisted of three sectors-the left semicircle (signal), which was the particle accumulation zone, the upper quartile (clean), which was the maximum intensity/chromatic reference zone and the lower quartile (x0.5ND) which gave a second level of light intensity/chromatic referencing. During a monitoring operation, the authenticity of a chromatic signal was checked against the invariant $\mathrm{H}, \mathrm{L}, \mathrm{S}$ values of the reference quartiles (clean, $\mathrm{x} 0.5 \mathrm{ND}$ ). Changes in particle concentrations were obtained using calibration curves of particle concentration versus chromatic L of the signal sector of the image [10]. Calibration procedures involved measuring changes in the chromatic parameters $(\mathrm{H}, \mathrm{L}, \mathrm{S})$ of light forward scattered by known concentrations of 2-10 micron particles accumulated on micropore particle filters which were also measured with a TEOM system at a statutory monitoring station (Sefton Borough Council). Measurements were repeated several times and with different optical intensities of the light source. As a result, a family of calibration curves of chromatic $\mathrm{L}$ 


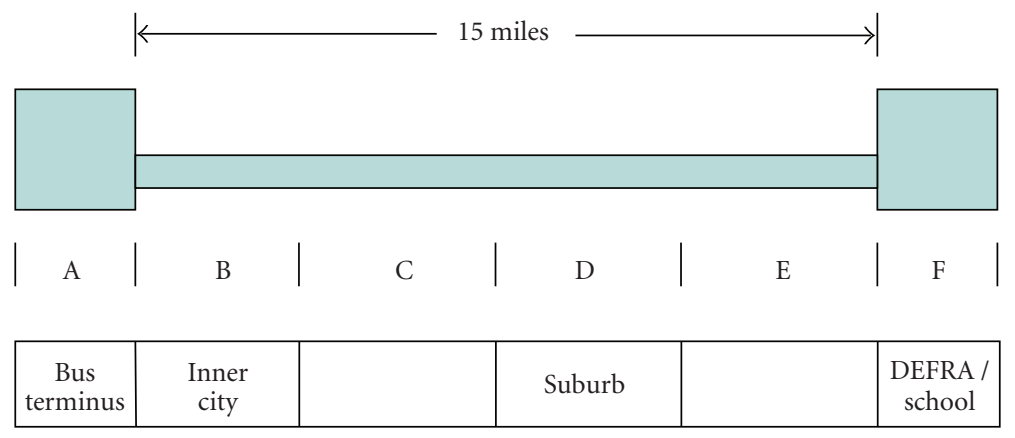

Figure 2: Schematic Diagram showing Sectors (A, B, C, D, E, F) of the Bus Corridor (A, Bus Terminus, B, Inner City, C, Main Traffic Artery, D, Suburban Dual Carriageway, E, Semi-industrial Dual Carriageway, F, School / DEFRA).

versus concentration were obtained (Figure 5, Reichelt et al. [10]). Chromatic $\mathrm{S}$ and $\mathrm{H}$ were used for providing additional information about the particle encoded scattered light with the possibility for some preliminary discrimination between certain particle types [12].

\section{Test Conditions}

The portable unit was assessed for providing holistic information about the occurrence of 2-10 micron airborne particles at a range of locations, under various conditions and at a height above ground (i.e., a few feet) at which the particles might be ingested by human beings. It was intended to identify areas and conditions showing higher levels of such particulates so that they could, if required, be retrospectively and more economically investigated using followup detailed measurements with precision instruments. Following laboratory and ideal site testing as described by Reichelt et al. [10], the performance and robustness of the monitoring unit were evaluated by testing at various sites along a fifteen mile long, urban bus corridor, on the arrival/departure apron of a busy city centre bus terminus and outside a local school adjacent to a suburban DEFRA air quality monitoring station.

The bus corridor over which tests were performed (Figure 2) extended from the city centre bus terminus to a location close to the regulatory (DEFRA) air quality monitoring station. The corridor included some inner city areas, a suburban area with low traffic density and a semi industrial area. The corridor was divided into six sectors (A, B, C, D, E, F) corresponding to such different regions. Conditions were monitored during both outward and return journeys, the time duration of each being approximately 45 minutes. Measurements were made with the vehicle stationary at locations within each sector in order to minimise any extraneous effects due to the vehicle's own exhaust, movement, and so forth and with the monitoring unit a few feet above the kerbside. Ten tests were undertaken over a period of about two months and on the same two afternoons of each week. Detailed records were kept of the various conditions under which each test was undertaken, for example temperature, humidity levels, traffic density, time at which the vehicle was at various locations, and so forth. The drive voltage of the optical source was adjusted and recorded to ensure that the particle monitor operated within an appropriate calibration range [10]. Inclusion of the DEFRA site within the extensive length of the bus corridor was significant in that it provided a means for conveniently benchmarking the operation of the chromatic portable unit during testing.

A further set of tests was undertaken monitoring particulates levels on an arrival/departure apron at the city centre bus terminus. During the testing the arrival, temporary parking, departure and transit of various buses, some with and others without particle traps fitted on their exhausts occurred. Several monitoring sessions were undertaken lasting typically for one hour each. During such sessions, a diary was kept recording the arrival, duration of stay, and departure of individual buses and whether they had particle filters fitted. Also visual observations of any excessive particles emissions were made.

\section{Test Results}

Bearing in mind the holistic nature of the testing, the results are in the form of trends in the occurrence of 2-10 micron particle concentrations rather than high accuracy, detailed measurements. Therefore the results are presented in terms of variations in concentrations over various time scales (ranging from a few minutes to several weeks), the number of sites addressed with the single unit, response to perturbing events (e.g., number of buses without particle traps on the bus station apron at given times), and so forth. Typical results obtained from tests with the portable chromatic monitoring unit at sites along the urban bus corridor and at the bus terminus are shown on Figures 3 and 4, respectively.

4.1. Urban Bus Corridor. Results obtained from monitoring sites along the 15 mile long bus corridor (Figure 3) are in the form of the chromatic parameter $\mathrm{L}$ as a function of time. For the test shown, the value of $\mathrm{L}$ reduced from about 0.8 at the commencement of the test to 0.62 at the end, well within the acceptable operational range (0.050.95 [10]). Transformation of the $L$ values into accumulated particle concentrations was achieved using the calibration data relating $\mathrm{L}$ to particle concentration with source intensity 


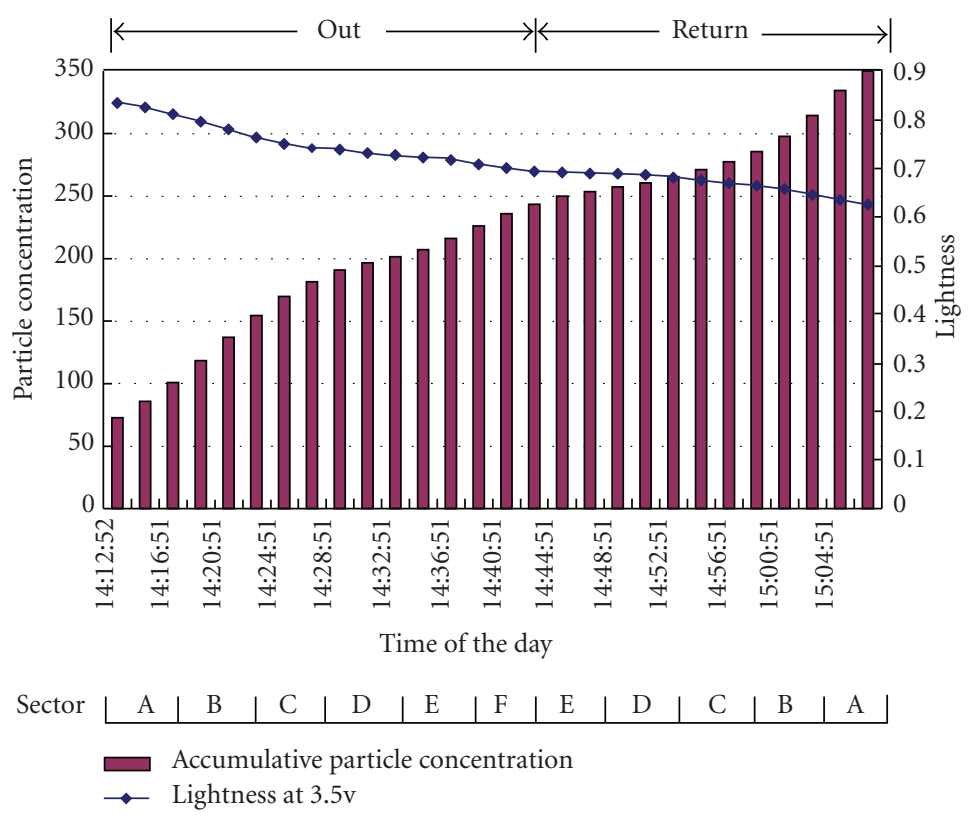

FIgURE 3: Time variation of the chromatic parameter $\mathrm{L}$ and derived accumulated particle concentration along the urban bus corridor (outward and return journeys).

as parameter (Figure 5, [10]). This enabled the time variation of the accumulated particle concentrations to be obtained from the time variation of $\mathrm{L}$. Both the time variation of $\mathrm{L}$ and the resulting particle concentration variation determined from the $\mathrm{L}$ values for one test are shown on Figure 3.

Since the times correspond to different locations along the bus corridor, the results on Figure 3 also indicate the accumulated particle concentrations within each of the location sectors (A, B, C, D, E, F (Figure 2)). It should be noted that both outward and return journeys are included along the time axis so that each sector appears twice, the second time in reverse order, corresponding to the return journey. The figure shows that the rate of increase of the accumulated particle concentration is non-uniform with respect, not only to time but also the contributions made by the various sector locations.

4.2. Bus Terminus. Figure 4(a) shows results of one test at the city centre bus terminus in the form of the time variation of the three chromatic parameters $H, L, S$. Values for each of these parameters are shown every ten minutes over a period of 60 minutes with an intake air flow into the monitoring unit of $10 \mathrm{l} / \mathrm{m}$. The $\mathrm{L}$ parameter values reduced monotonically from 0.77 to 0.27 whilst the $\mathrm{S}$ parameter values remained within the range 0.05 to 0.1 . The $\mathrm{H}$ parameter showed an increase from an initial value of 40 degrees to about 48 degrees before reverting to a value of 40 degrees.

Figure 4(b) shows the particulates concentration accumulated over the 10 minutes duration of each of the six time windows of Figure 4(a) and determined from the $\mathrm{L}$ parameter changes using the calibration curves presented by Reichelt et al. [10]. (Also shown on Figure 4(b) is the L parameter variation.) The results show irregular variations of concentration between the six time windows.

During the period of this test a total of 24 buses arrived and departed from the bus apron with resident times at the apron of up to 5 minutes. Twelve of these buses had particle traps mounted on their exhausts and there were three traverses without stopping of one bus with a visibly higher emission of exhaust smoke. Records of such bus activities, and so forth. were kept. Figure 5 shows a graphical representation of the number of buses with and without a particle trap on the exhaust, and the duration of their presence, as a function of time within three 10 minutes time windows $(1,2,3)$ corresponding to the first three windows of Figure 4(b). Also shown are the times at which the high pollution bus passed the apron.

\section{Discussion of Results}

The results obtained from the various tests may be considered in terms of their holistic implications regarding broad variations of particle concentrations with time, location and specific events.

5.1. Bus Corridor Results. The accumulated particle concentrations as a function of time and sector $(A, B, C, D$, E, F) along the bus corridor shown on Figure 3 may be transformed into the particle concentration for each sector by subtracting the accumulated particle concentration of adjacent sectors from each other. The distribution of particle concentrations across the six sectors during one test sequence may then be obtained.

Figure 6 shows such a distribution of the relative particle concentrations over the six route sectors for 10 tests, each 
test result corresponding to a separate outward journey. Each sector on the horizontal scale contains the 10 test results for that sector in the order of the sequence of the tests. The diagram illustrates the complexity of the results but it is nonetheless possible to discriminate approximate trends by finding the averages of the relative concentrations over the ten tests for each sector. This shows that the relative particulates level averaged over 10 tests for sector F (a few feet above ground at the school entrance) was $40 \%$ higher than that for sector A (the bus terminus). The lowest level (2.15) was in sector D (quiet suburban area) being only $27 \%$ of the sector A (bus terminus) level. The holistic nature of these results encompasses variations in background effects such as variable weather conditions (including wind born micro-particles), diurnal effects and traffic density changes, and so forth.

The time periods during which measurements were made with the vehicle stationery within each location sector were noted and taken into account, but more precise estimates might be advisable in future tests. Small uncertainties in such time periods do not seem to account for any major differences in the particulates levels. For example, the time period ( 15.00-15.15 hours) over which the sector F (school entrance) values were obtained coincided with parental vehicles calling to collect school children which would accord with the consistent, relatively high levels recorded by the portable unit for sector F. These particle levels recorded by the portable unit could also be compared retrospectively with values from a TEOM monitor at the DEFRA air quality monitoring station adjacent to the school. Results of tests conducted with the portable unit over an extended time period, mid morning with only low level traffic activity at this site, showed reasonable agreement with values from the DEFRA TEOM unit [10] so confirming the correct operation of the portable unit. However, particulate levels measured with the portable chromatic unit in the presence of stationery vehicles around 15 hours, collecting children from the adjacent school, were about twice the levels from the DEFRA TEOM unit. This difference may be ascribed to the DEFRA unit sampling air about $4 \mathrm{~m}$ above ground level compared with the portable unit being about 4 feet above ground and the DEFRA unit values being integrated over a 30 -minute period which was not synchronised with the 15 minutes recording period of the portable unit.

5.2. Bus Terminus. Results of the form given on Figures 4 and 5 enable a preliminary comparison to be made between the concentration of airborne PM 2-10 particles at the apron of the bus terminus and the number and type of buses on the apron at a given time. Of course factors other than the bus population (e.g., weather conditions, etc.) can affect such a comparison, but the approach is warranted for providing preliminary indications of conditions requiring more detailed investigation with specialised precision instruments.

Such a comparison of particle concentration with bus population indicates that there appears to be a correlation between the number of buses without particle traps on their exhausts and the particulates levels indicated by the monitor. In addition, the passage of the bus with visibly higher

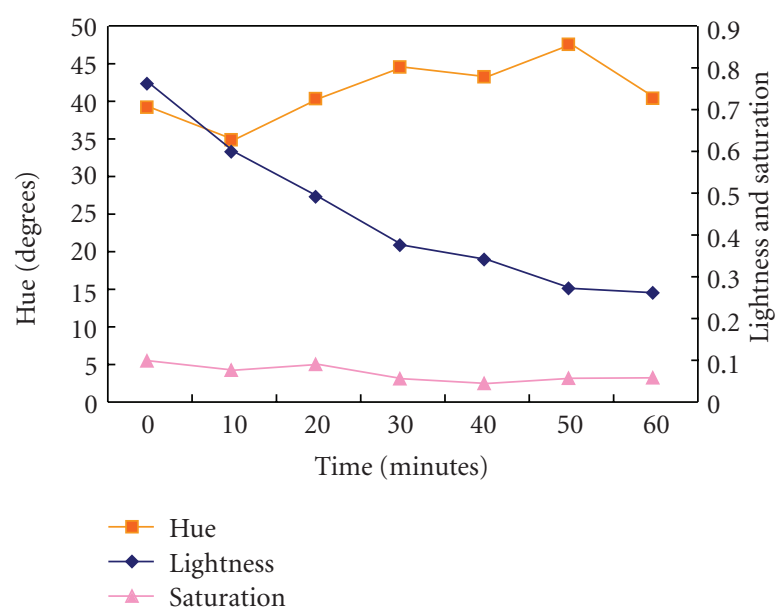

(a)

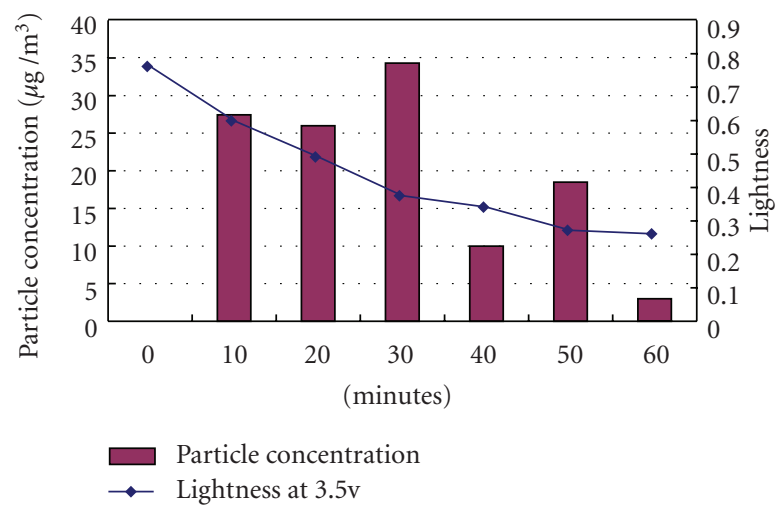

(b)

FIGURE 4: Time variation of chromatic parameters and particle concentration at the city centre bus terminus. (a) Chromatic Parameters H, L, S versus time, (b) L and derived particle concentration for each time window versus time.

smoke emission from its exhaust appeared to transiently increase the particle concentration indicated by the monitor by about a factor of two. Such results provide credence for the operation of the monitoring unit under robust real conditions, and suggest that this aspect of the bus terminus conditions warrants more detailed measurements with precision instruments.

A further aspect of the results obtained with the portable chromatic monitor on the bus apron concerns distinguishing between different types of particles (i.e., size, nature, etc.) from trends in a second chromatic parameter $(\mathrm{H})$ as well as L. Under most conditions investigated, the reduction in the value of chromatic $\mathrm{L}$ as the micro particles accumulated, was accompanied by a reduction in the value of $\mathrm{H}[10]-$ consistent with the long wavelength shift in forward scattered light produced by micro particles (Mie scattering [15]). However the results obtained during the presence of the high smoke emitting bus showed an increase in the value of $\mathrm{H}$ from 40 to 48 degrees ( $\mathrm{H}$ curve, Figure 4(a)), indicating a shift in dominant wavelength towards shorter rather than longer values. Such a shift is indicative of the presence of different types of particles (e.g., [16]). 


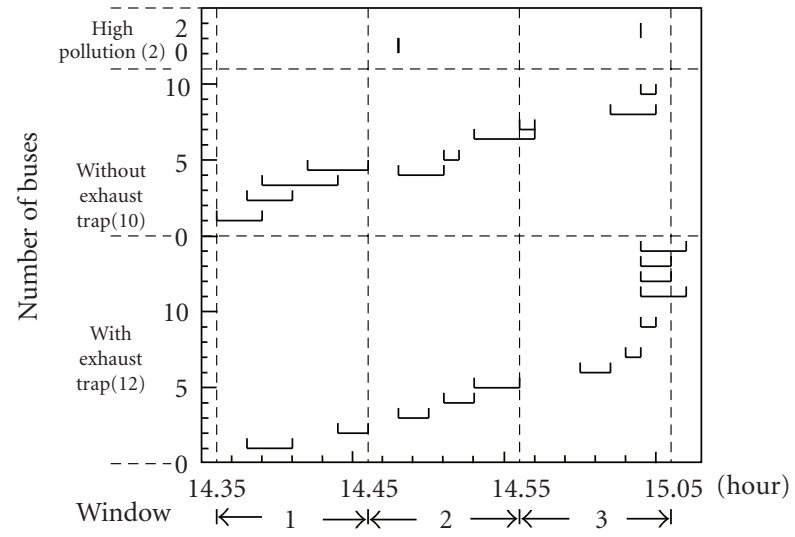

Figure 5: Sample Record of Different Bus Populations and Visit Durations as Functions of Time for Three 10 Minute Time Windows 1, 2, 3 .

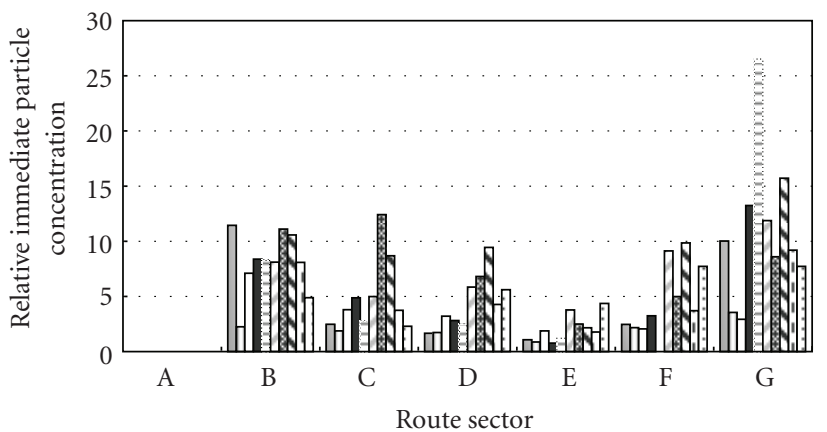

$\begin{array}{ll}\square \text { Micro156 021104 } & \square \text { Micro166 301104 } \\ \square \text { Micro158 041104 } & \square \text { Micro167 021204 } \\ \square \text { Micro162 181104 } & \square \text { Micro168 071204 } \\ \square \text { Micro164 231104 } & \square \text { Micro171 091204 } \\ \square \text { Micro165 251104 } & \square \text { Micro173 141204 }\end{array}$

Figure 6: Particle Concentrations for each of 10 Tests as Functions of Sectors (A, B, C, D, E, F) along the bus corridor (outward journeys).

Consequently, these results at the bus terminus apron show that the chromatic particle monitor can, not only indicate situations and events which might be associated with elevated particle concentration levels worthy of more detailed investigation with precision instruments, but can also highlight possible variations associated with the nature of the particles collected which would also warrant further investigation.

\section{Conclusions}

The portability and robustness of the chromatic particle monitor unit has been demonstrated for obtaining fingerprints of airborne PM10 particulates over widespread areas (e g. at sites along a 15 mile long urban bus corridor) and under difficult environmental conditions (e.g., apron of a busy bus terminus). It has been shown that the unit has made it possible for variations of PM 2-10 particle concentrations to be conveniently and economically obtained at various sites along an urban bus corridor at different times, dates, and so forth. Such results included data gathered under different environmental conditions (weather, wind borne dust particles, etc.) which can be further analysed to gain an insight into such effects using discrete chromatic processing as described by Deakin et al. [16].

It has also been shown that the results obtained with the chromatic monitoring unit can, not only be conveniently benchmarked during field use (e.g., against a particle monitor (TEOM) at a DEFRA air quality monitoring station), but can also highlight situations (e.g., school collection traffic) which warrant more detailed investigation with precision, particle specific measurement instruments.

The results of tests at the city centre bus terminus have shown that the portable monitor was sufficiently robust for such deployment. They have also shown that, in such an environment, the monitor was capable of not only indicating conditions/events worthy of more detailed investigation with precision instruments, but could provide some additional information from a second chromatic parameter $(\mathrm{H})$, regarding possible changes in particle types which would be worth addressing in a more rigorous manner.

Although the potential of the portable monitoring unit for providing signatures of airborne particulates under onerous site conditions has been demonstrated, the particulates levels quoted in this contribution should not be regarded as formally authenticated. Nonetheless it demonstrates the potential of the approach for providing preliminary field assessments before deploying more expensive and time consuming, precision, and specialised instrumentation. Further development of the approach is warranted and is currently ongoing to produce a more compact unit with onboard processing, and so forth.

\section{Appendix}

Chromaticity parameter L, S, H are defined as follows:

$$
\begin{gathered}
L=\frac{(R+B+G)}{3}, \\
S=\frac{\max (R, B, G)-\min (R, B, G)}{\max (R, B, G)+\min (R, B, G)}, \\
H= \begin{cases}\frac{60(G-B)}{(\max (R, G, B)-\min (R, G, B))} & \text { if } \max (R, G, B)=R, \\
\frac{60(2+(B-R))}{(\max (R, G, B)-\min (R, G, B))} & \text { if } \max (R, G, B)=G, \\
\frac{60(4+(R-G))}{(\max (R, G, B)-\min (R, G, B))} & \text { if } \max (R, G, B)=B,\end{cases}
\end{gathered}
$$

where $R, G, B$ are the outputs from each of the three detectors of the optoelectronic array. $\operatorname{Max}(R, G, B)$ and $\min (R, G, B)$ refer to the channel having the highest and lowest output values, respectively. 


\section{Acknowledgments}

The support and cooperation of the following organizations during the pursuit of this work is acknowledged: Liverpool City Council, Merseytravel, Arriva, Sefton Borough Council, TTR, the EU Life Project, and the EU ERDF which enabled the instrumentation to be developed.

\section{References}

[1] E. Vega, E. Reyes, G. Sanchez, et al., "Basic statistics of PM2.5 and PM10 in the atmosphere of Mexico City," The Science of the Total Environment, vol. 287, pp. 167-176, 2002.

[2] X. Querol, A. Alastvey, C. R. Ruiz, et al., "Speciation and origin of PM10 and PM 2.5 in selected European cities," Atmospheric Environment, vol. 38, pp. 6547-6555, 2004.

[3] G. W. Fuller and D. Green, "The impact of local fugitive PM10 from building works land road works on the assessment of the European Union Limit Value," Atmospheric Environment, vol. 38, pp. 4493-5002, 2004.

[4] P. Lenschow, H.-J. Abraham, K. Kutzner, M. Lutz, J.-D. Preuß, and W. Reichenbächer, "Some ideas about the sources of PM10," Atmospheric Environment, vol. 35, supplement 1, pp. S23-S33, 2001.

[5] R. M. Harrison, "Studies of the source apportionment of airborne particulate matter in the United Kingdom," Journal of Aerosol Science, vol. 31, supplement 1, pp. S106-S107, 2000.

[6] J. R. Pfaffin and E. N. Zeigler, Encyclopedia of Environmental Science and Engineering, vol. 2, Taylor \& Francis/CRC Press, Boca Raton, Fla, USA, 5th edition, 2006.

[7] M. B. Meyer, H. Patashnick, J. L. Ambs, and E. Rupprecht, "Development of a simple equilibration system for the TEOM continuous PM monitor," Journal of Air and Waste Management Association, 2000.

[8] C. S. Johnson and D. A. Gabriel, Laser Light Scattering, Dover, New York, NY, USA, 1981.

[9] R. Hitzengerger, S. G. Jennings, S. M. Larson, et al., "Intercomparison of measurement methods for Black Aerosols," Atmospheric Environment, vol. 33, no. 17, pp. 2823-2833, 1999.

[10] T. E. Reichelt, M. A. Aceves-Fernandez, Y. R. Kolupula, A. Pate, J. W. Spencer, and G. R. Jones, "Chromatic modulation monitoring of airborne particulates," Measurement Science and Technology, vol. 17, no. 4, pp. 675-683, 2006.

[11] G. R. Jones, P. C. Russell, A. Vourdas, J. Cosgrave, L. Stergioulas, and R. Haber, "The Gabor transform basis of chromatic monitoring," Measurement Science and Technology, vol. 11, no. 5, pp. 489-498, 2000.

[12] G. R. Jones, A. G. Deakin, and J. W. Spencer, Chromatic Monitoring of Complex Conditions, Taylor \& Francis, Boca Raton, Fla, USA, 2008.

[13] M. W. Schwarz, W. B. Cowan, and J. C. Beatty, "An experimental comparison of RGB, YIQ, LAB, HSV and opponent colour models," ACM Transactions on Graphics, vol. 6, pp. 123-158, 1987.

[14] H. Levkowitz and G. T. Herman, "GLHS: a generalized lightness, hue, and saturation color model," Graphical Models and Image Processing, vol. 55, no. 4, pp. 271-285, 1993.

[15] M. Kerker, The Scattering of Light and Other Electromagnetic Radiation, Academic Press, New York, NY, USA, 1969.
[16] A. G. Deakin, Y. R. Kolupula, A. Koh, H. M. Looe, and J. Zhang, "Characteristics of chromatic H, L, S systems," in Chromatic Monitoring of Complex Conditions, G. R. Jones, A. G. Deakin, and J. W. Spencer, Eds., chapter 2, section 2.6, Taylor \& Francis, Boca Raton, Fla, USA, 2008. 

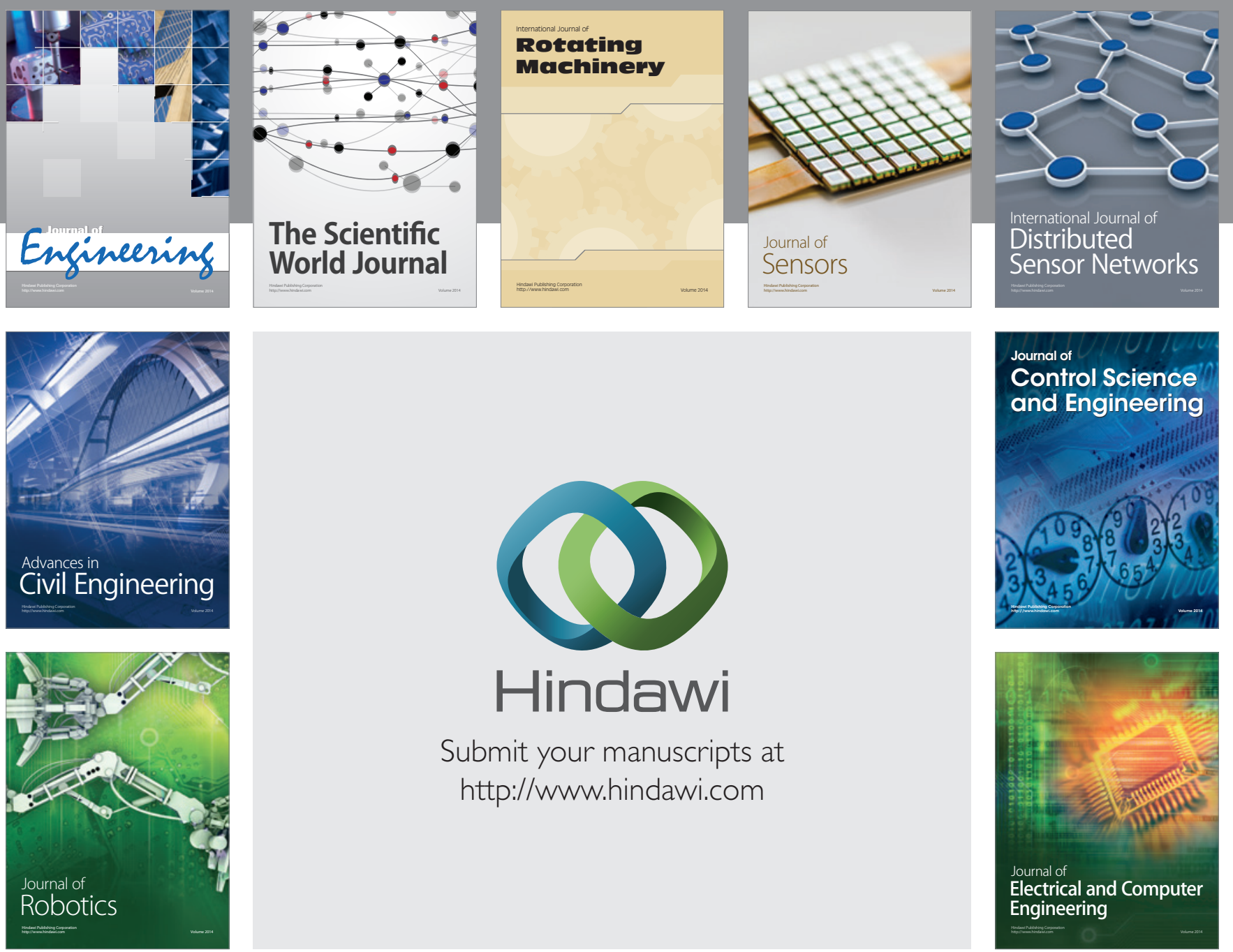

Submit your manuscripts at

http://www.hindawi.com
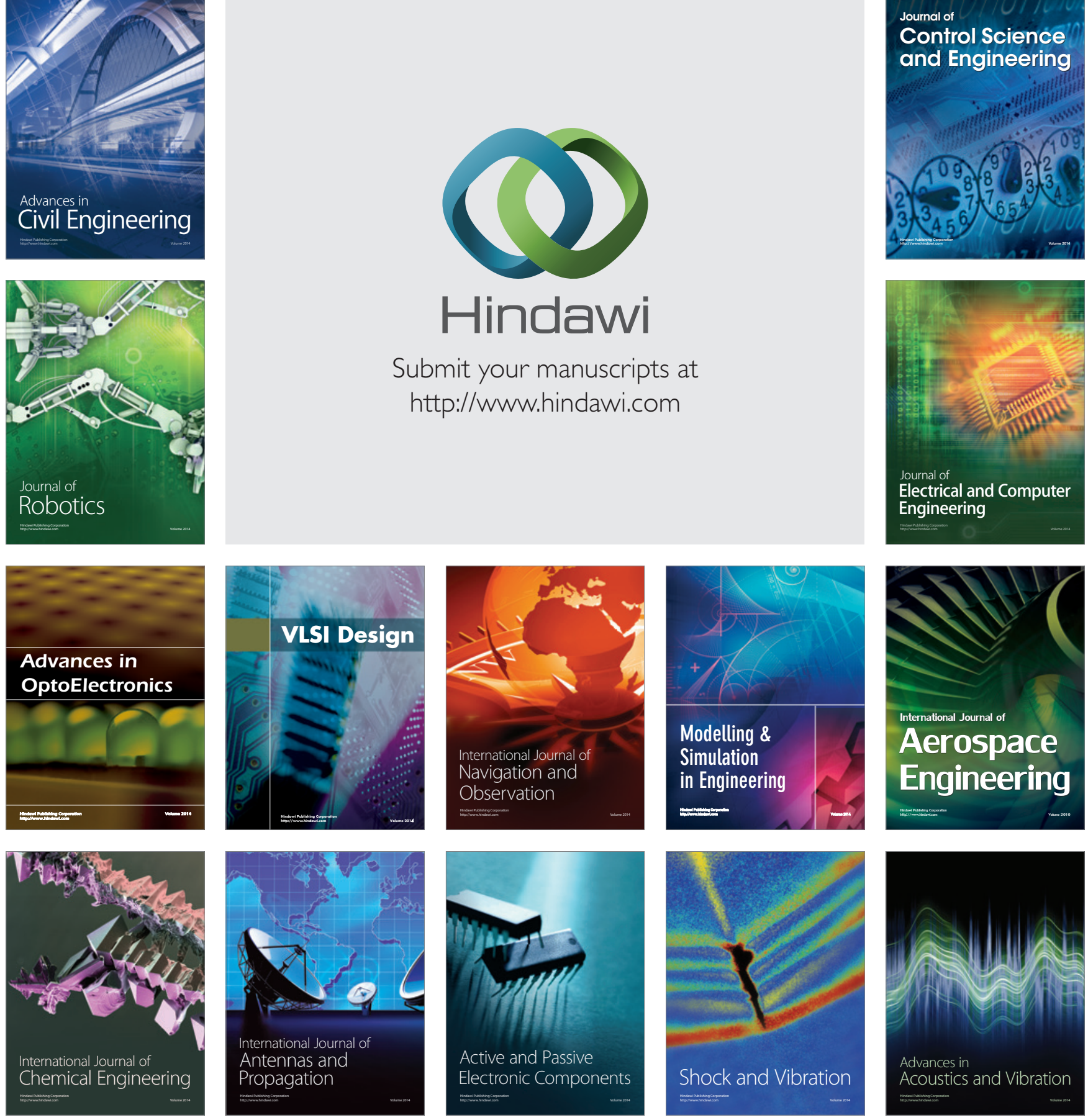